University of Wollongong

Research Online

Faculty of Engineering and Information

Faculty of Engineering and Information

Sciences - Papers: Part A

Sciences

2014

\title{
A Novel Proton Pencil Beam Scanning Technique for Postmastectomy Chest Wall Irradiation
}

\author{
Nicolas Depauw \\ University of Wollongong, nd999@uowmail.edu.au \\ E Batin \\ Massachusetts General Hospital \\ J Daartz \\ Massachusetts General Hospital \\ S Macdonald \\ Massachusetts General Hospital \\ Hanne Kooy \\ Massachusetts General Hospital
}

See next page for additional authors

Follow this and additional works at: https://ro.uow.edu.au/eispapers

Part of the Engineering Commons, and the Science and Technology Studies Commons

Research Online is the open access institutional repository for the University of Wollongong. For further information contact the UOW Library: research-pubs@uow.edu.au 


\title{
A Novel Proton Pencil Beam Scanning Technique for Postmastectomy Chest Wall Irradiation
}

\author{
Abstract \\ Abstract of paper presented at the American Society for Radiation Oncology 56th Annual Meeting, San \\ Francisco, United States, 14 - 17 September 2014. \\ Keywords \\ novel, proton, pencil, technique, postmastectomy, scanning, chest, wall, beam, irradiation \\ Disciplines \\ Engineering | Science and Technology Studies \\ Publication Details \\ Depauw, N., Batin, E., Daartz, J., Macdonald, S., Kooy, H. \& Lu, H. (2014). A Novel Proton Pencil Beam \\ Scanning Technique for Postmastectomy Chest Wall Irradiation. International Journal of Radiation: \\ Oncology - Biology - Physics, 90 (1, Suppl. 1), S932-S932. \\ Authors \\ Nicolas Depauw, E Batin, J Daartz, S Macdonald, Hanne Kooy, and Hsaio-Ming Lu
}




\section{A Novel Proton Pencil Beam Scanning Technique for Postmastectomy Chest Wall Irradiation}

N. Depauw,1,2 E. Batin,1 J. Daartz,1 S. MacDonald,1 H. Kooy,1 and H. Lu1; 1Massachusetts General Hospital, Boston, MA, 2University of Wollongong, Wollongong, Australia

Purpose/Objective(s): Post-mastectomy radiation therapy treatment (PMRT) is conventionally performed using a composition of photon and electron fields. Including the internal mammary nodes is always a challenge in such treatment for patients with unfavorable cardiac anatomy and/or breast implants. Furthermore, multi-field matching results in hot and cold spots, as well as extended treatment time. We have developed a PMRT technique using a single proton pencil beam scanning (PBS) field that includes the whole chest wall and all nodal regions with complete target coverage and reduced dose to cardiac and lung tissues.

Materials/Methods: The patients undergo a CT scan on an angled breast board, as for regular photon/electron treatment, but with the addition of a chin strap and hand grips to provide additional immobilization and reproducibility. An in-house software, with multicriteria optimization (MCO) capability, is used for PBS treatment planning. A single isocentric field, angled at 30 degrees from vertical, is used to cover the whole chest wall and the potentially involved lymph nodes (axillary, supraclavicular, internal mammary), all of which volumes are combined into a single scanning target volume (STV) for the spot map optimization. An $8 \mathrm{~cm}$ thick range shifter, made of Lucite, is used to reduce the proton beam energy in order to appropriately cover the superficial portion of the target volumes. The PBS field usually contains less than 15 layers equally spaced about $5 \mathrm{~mm}$, with a total number of spots in the range of 1500 to 2000 . For patients with breast implants, the stopping power ratio of the implant materials was separately measured, and the remaining range uncertainty is limited to that from the chest wall only. Surface imaging, along with daily radiography, is used for patient positioning in order to improve setup accuracy for the largely superficial target volume and to monitor anatomical changes during the course of treatment.

Results: Over 20 patients (mostly left sided) have been treated at the institution using the described technique. A single PBS field proves to be sufficient to deliver full and more homogeneous dose coverage of all the target volumes as well as less dose to cardiac tissues compared to conventional photon/electron treatment plans. All treatment fields pass QA gamma test by more than $95 \%$ ( $3 \mathrm{~mm} / 3 \%$ of prescription dose). A planning study, based on 4D CT, showed that the PBS dose distribution is robust to patient's breathing, as well as to patient's setup uncertainties, $3 \mathrm{~mm}$ shifts and $2^{\circ}$ rotations.

Conclusions: A novel treatment technique for post-mastectomy chest wall irradiation using proton PBS fields is currently offered by the institution. While improving both target coverage and OAR sparing, this technique also offers simpler planning and reduced treatment time. 